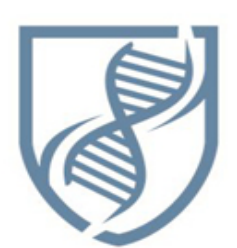

Journal of Bioscience and Applied Research
JBAAR

WWW.JBAAR.ORG

\title{
Protective effect of Coenzyme Q10 against gentamicin induced acute renal failure in mice
}

\author{
Abdel Razik H. Farrag ${ }^{1}$, Rania A. Ibrahim², Shimaa N. El-Sayed ${ }^{2}$ \\ ${ }^{1}$ Pathology Department, Medical Research Division, National Research Centre, Cairo, Egypt. \\ ${ }^{2}$ Zoology Department, Faculty of Science, Suez University, Suez, Egypt.
}

(Corresponding author e-mail. rania.sheir@yahoo.com)

\begin{abstract}
Gentamicin is an antibiotic whose clinical use is limited by its nephrotoxicity. Coenzyme Q10 (CoQ10) is an effective antioxidant and used for therapy of a number of diseases. The present study was designed to investigate the possible protective effect of CoQ10 against gentamicin induced nephrotoxicity in mice. Thirty five adult male mice were used in this study and were randomly divided into five groups, each consisting of seven animals as follows: group I: normal control; group II: treated with CoQ10 (30 $\mathrm{mg} / \mathrm{kg} /$ day, orally for 14 days); group III: treated with gentamicin $(80 \mathrm{mg} / \mathrm{kg} / \mathrm{day}$, i.p. for 14 days); group IV: treated with CoQ10 and gentamicin for 14 days; group V: treated with gentamicin $(80 \mathrm{mg} / \mathrm{kg} /$ day) i.p. for 14 days, after that the animals were given CoQ10 (30 mg/kg/day) orally for 7 days. At the end of the experiment, blood was collected and serum was separated for the estimation of serum creatinine and urea. Then the mice were sacrificed and kidneys were removed for histopathological study. The biochemical results showed that Coenzyme Q10 administration with gentamicin injections significantly decreased serum urea and creatinine when compared with gentamicin group. Light microscopic examination of the renal tissues from gentamicin-treated mice revealed severe histopathological changes, whereas specimens obtained from CoQ10 treated mice revealed only mild changes. Conclusion: It appears that CoQ10 has some protective role against gentamicin induced nephrotoxicity.
\end{abstract}

Keywords: Gentamicin, Coenzyme Q10, nephrotoxicity, histopathology, mice.

\section{Introduction}

Gentamicin is one of the most commonly used aminoglycoside antibiotics for the treatment of serious and life-threatening infections caused by Gram-negative aerobes (Negrette-Guzman et al., 2013). Despite its beneficial effects, low cost and low levels of resistance, serious complications like nephrotoxicity and ototoxicity are dose-limiting factors in the use of aminoglycosides (Sun et al., 2013). This antibiotic generally causes druginduced dose-dependent nephrotoxicity in 10-20\% of therapeutic courses (Martínez-Salgado et al., 2007).The toxicity of gentamicin seems to relate to the generation of destructive reactive oxygen species (ROS) in these cells (Al-Majed et al., 2002; Reiter et al., 2002). ROS have been proposed as a causative agent of cell death in many different pathological states (Mizrak et al., 2004) including glomerular disease (Cuzzocrea et al., 2002) and renal ischemia reperfusion injury (Sahna et al., 2003). Most research studies against gentamicin-induced nephrotoxicity are focused on the use of various antioxidants (Khan et al., 2009).

Coenzyme Q10, also known as ubiquinone, is an naturally-occurring lipid soluble antioxidant that is endogenously synthesized (Littarru and Tiano, 2007). It is used as a dietary supplementation and as a co therapy in conjunction with medication in a number of conditions, including cardiovascular diseases, cancer, muscular neurodegenerative disorders, and diabetes (Villalba et al., 2010). The nephroprotective effect of CoQ10 is still controversial. On one hand, CoQ10 showed nephroprotective effects in some animal models (Fouad et al., 2010; Persson et al., 2012). On the other hand, no renal 
protection has been reported in another animal study (Sutken et al., 2007). So this work was designed to study the protective effect of the CoQ10 on gentamicin-induced renal damage.

\section{Materials and Methods}

\subsection{Chemicals:}

Coenzyme Q10 was produced by Arab Company for Pharmaceuticals and Medical Plants (MEPACO MEDIFOOD), Enshas El Raml, Sharkeya, Egypt. The dose of CoQ10 was $30 \mathrm{mg} / \mathrm{kg}$ body weight. CoQ10 was dissolved in distilled water and administered orally every day. Gentamicin sulphate (Garamicin $80 \mathrm{mg} / 2 \mathrm{ml}$ ampoule) was purchased from Memphis Co. for Pharm. \& Chem.ind. Cairo- A.R.E. under authority of Schering Plough Coporation/U.S.A. The dose of gentamicin was 80 $\mathrm{mg} / \mathrm{kg}$ body weight and was given intraperitoneally for consecutive 14 days.

\subsection{Animals}

Male albino mice obtained from Laboratory Animal House, National Research Centre, Cairo, Egypt,weighing 20 - 25 g were used in this study. The animals were housed in groups of 7 in stainless steel community cages at $22 \pm 2$ ${ }^{\circ} \mathrm{C}$ with a $12 \mathrm{hr}$. light/dark cycle and allowed to acclimatize for one week prior to experimental use. Throughout the experiment, the mice allowed free access feed (mice dietary pellets prepared by Cairo Company of Oil \& Soap, Egypt) and water was allowed ad libitum.

\subsection{Experimental Design:}

Thirty five mice were used and classified into 5 groups (7 mice / group).

\section{Group I: The control group.}

Group II: Mice were given CoQ10 (30 mg/kg b.w.) orally for 14 days.

Group III: Mice were given gentamicin (80 mg/kg b.w.) intraperitoneally for 14 days.

Group IV: Mice were given CoQ10 (30 mg/kg b.w.) orally 3 hours before giving gentamicin ( $80 \mathrm{mg} / \mathrm{kg} \mathrm{b.w.)} \mathrm{for} 14$ days.

Group V: Mice were given gentamicin (80 mg/kg b.w.) intraperitoneally for 14 days, after that the animals were given CoQ10 (30 mg/kg b.w.) orally for 7 days.

\subsection{Determination of serum creatinine and urea levels:}

Blood samples were collected using the orbital sinus technique of Sanford (1954). Blood was collected in dry tube and left to clot, then centrifuged at 5000 r.p.m for ten minutes. Serum was separated and stored at $-20^{\circ} \mathrm{C}$ for subsequent measurement of serum creatinine and urea levels. The serum parameters were analyzed spectrophotometrically by using double beam UV-Visible spectrophotometer. Estimation of serum creatinine and urea were carried out using olorimetric assay kits from Vitroscient, Egypt according to the methods of Faulkner and King (1976) and Patton and Crouch (1977) respectively.

\subsection{Histopathological method:}

Mice were sacrificed and their kidneys were removed and prepared for histopathological examination Kidneys were dissected out and fixed instantaneously in $10 \%$ formal saline for 24 hours. The specimens were washed in tap water, dehydrated in ascending grades of ethanol, cleared in xylene, embedded in paraffin wax (melting point $55-60{ }^{\circ} \mathrm{C}$ ). Paraffin sections of $6 \mu \mathrm{m}$ thicknesses were prepared and stained with Haematoxylin and Eosin (H and E) (Drury and Wallington, 1980).

\subsection{Statistical analysis}

Microsoft Excel 2007 and SPSS (version 21.0) software programs were used for data analysis. Results were expressed as the means \pm SEM. Statistical significant difference was determined by one-way analysis of variance (ANOVA) followed by post hoc Tukey test for multiple comparison. Probability values $(P)$ less than 0.05 were considered to be statistically significant.

\section{Results}

\subsection{Bicochemical results}

Administration of CoQ10 alone had no effect on the level of serum creatinine and urea. Gentamicin treated group showed a significant increase at $p<0.05$ in the level of serum creatinine and urea as compared to the control group. On the other hand, gentamicin pre-treatment with CoQ10 showed a significant reduction at $\mathrm{P}<0.01$ in the high levels of serum creatinine and urea as compared with gentamicin group. Gentamicin post-treatment with CoQ10 resulted in significant decrease at $\mathrm{P}<0.01$ in the levels of serum creatinine and urea as compared with gentamicin group (Figure 1, a\&b)].

\subsection{Histopathological observations}

Microscopic examination of kidney sections of control mouse showed the normal structure of the renal corpuscles and tubules (Figure 2a). Examination of kidney sections of CoQ10 treated mice showed the normal structure of the renal corpuscles and tubules (Figure 2b). Microscopic examination of renal cortex of gentamicin treated mice showed atrophy in glomeruli, widening in Bowman's capsule, tubular dilatation and desquamation of their lining cells (Figure 2c). Interstitial hemorrhage was also shown (Figure 2c). Necrotic tubules with dense cytoplasm and pyknotic nuclei and hyaline material were observed (Figure 2d). There were congested blood vessels and some tubules showed complete loss of their lining cells (Figure 2d). These changes were most prominent in the proximal convoluted tubules. Cellular infiltration and hyaline degeneration were also showed (Figure 2e). Histopathological Examination of kidney sections of mice treated with CoQ10 and gentamicin showed the renal corpuscles and tubules appeared more or less like normal (Figure 2f). Examination of kidney sections of mice treated with gentamicin and CoQ10 showed the normal structure of the renal corpuscles and tubules. Some tubules showed vacuolar degeneration (Figure 2g). 


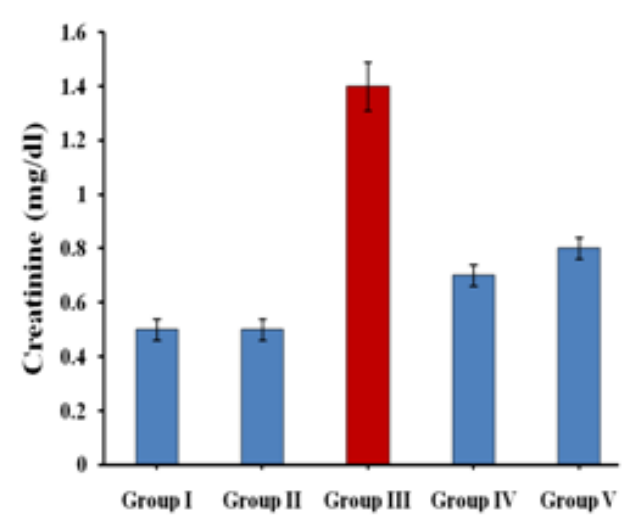

(a)

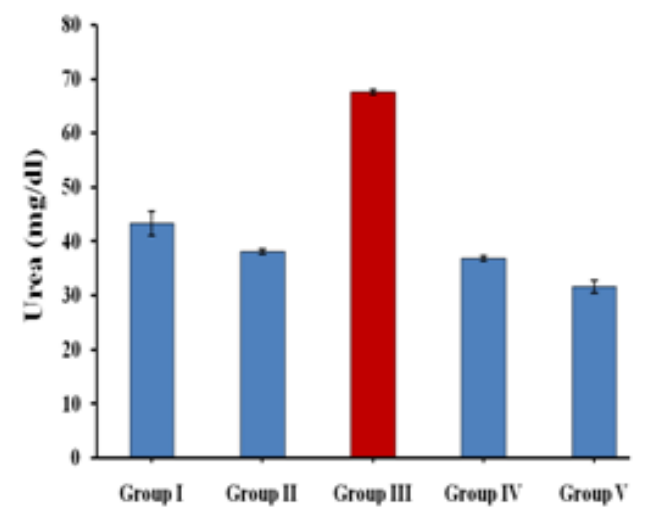

(b)

Figure (1): Effect of CoQ10 on serum creatinine (a) and urea (b) in mice treated with gentamicin.

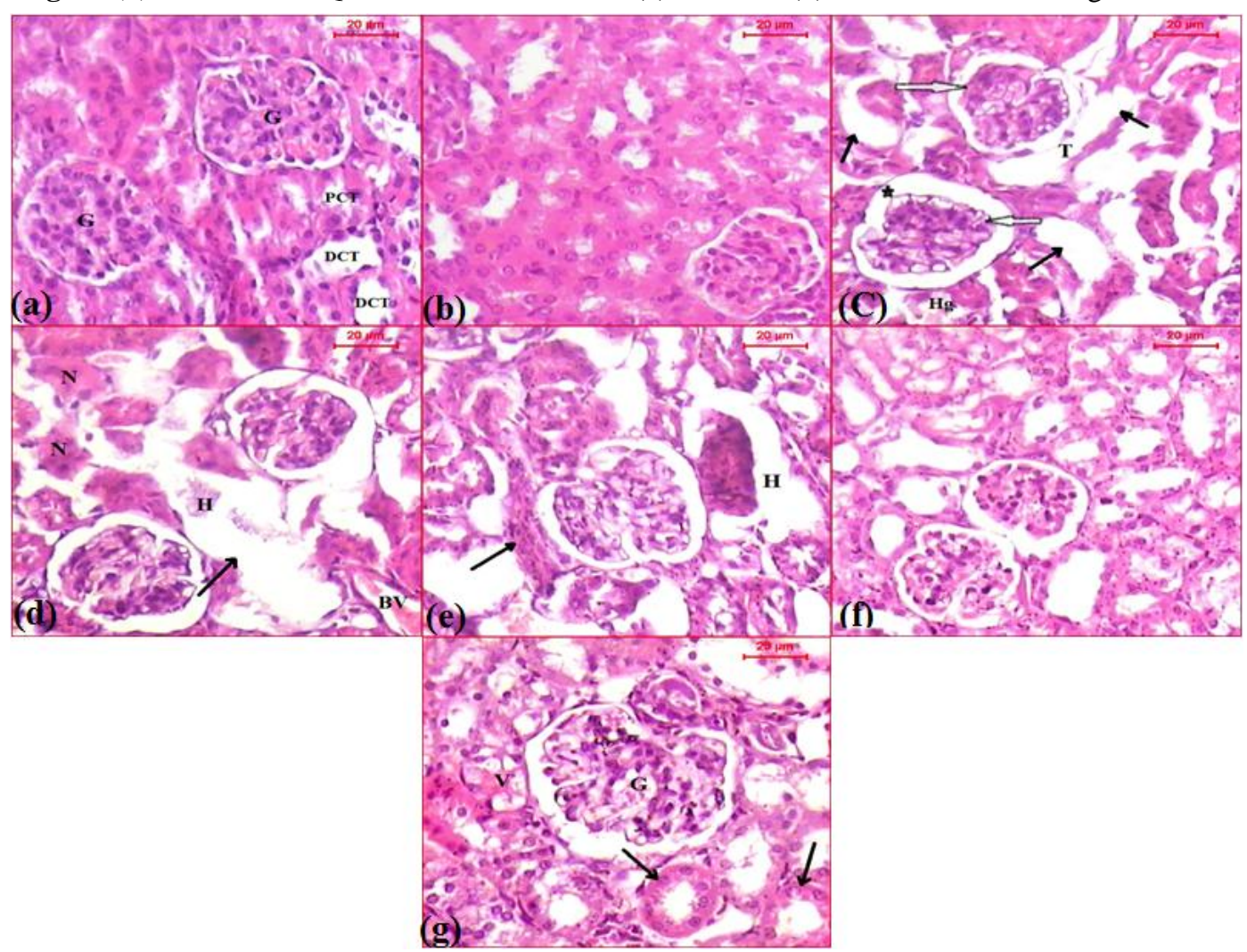

Figiure (2): Light microscope photomicrographs showing the protective effects of CoQ10 against histopathological alterations induced by gentamicin in the kidney tissues of different experimental groups (H\&E stain, scale bar: $20 \boldsymbol{\mu m})$. (a) Section from control group showing a glomerulus $(G)$ surrounded by proximal and distal convoluted tubules (PCTs and DCTs). (b) Section from CoQ10 treated group showing the normal structure of the renal corpuscle and tubules. (c) Section from gentamicin treated group showing hypotrophy in glomeruli (white arrows), widening in Bowman's space (star) and tubular dilatation (T) and desquamation of their lining cells (arrows). Notice: interstitial hemorrhage (Hg). (d) Section from gentamicin treated group showing tubular necrosis $(\mathrm{N})$ and presence of hyaline material $(\mathrm{H})$. Some tubules show complete loss of the lining cells in some tubules (arrow). Notice: congested blood vessel (BV). (e) Section from gentamicin treated mouse showing cellular infiltration (arrow) and hyaline degeneration (H). (f) CoQ10 and gentamicin treated mouse showing the renal corpuscles and tubules appeared more or less like normal. (g) Section from gentamicin and CoQ10 treated mouse showing the normal structure of the renal glomerulus (G) and tubules (arrows). Some tubules show vacuolar degeneration (V). 


\section{Discussion}

The aminoglycoside antibiotic gentamicin is an important agent against life threatening infections. However, nephrotoxicity is a major complication of the gentamicin administration. Thus amelioration of nephrotoxicity would enhance its clinical use. Several approaches involving the use of chemical compounds have been used to reduce gentamicin nephrotoxicity (Cuzzocrea et al., 2002; Parlakpinar et al., 2005). But little attention has been paid on the use of naturally occurring substances with potent antioxidant properties to protect against nephrotoxic damage induced by gentamicin. In the light of this, we have explored the possible protective role of CoQ10, a natural antioxidant substance on gentamicin-induced nephrotoxicity.

In the present work, serum creatinine and urea concentrations were estimated to assess the extent of nephrotoxicity. gentamicin treated mice showed a highly significant increase of serum creatinine and urea. This is confirmed with Karadeniz et al. (2008) and Moreira et al. (2014). The authors considered this increase as a result of nephrotoxicity. This observation was also reported by Farombi and Ekor (2006) who found that treatment with gentamicin (100 mg/kg, intraperitoneally) for 7 days resulted in significant elevations in serum urea and creatinine levels.

The current study showed that marked elevations of serum creatinine and urea concentrations were suggested as a significant functional impairment of kidney in gentamicin induced nephrotoxicity as previously explained by kalayarasan et al. (2009). Kumar et al. (2000) reported that administration of gentamicin (80 $\mathrm{mg} / \mathrm{kg} \quad$ b.w.) intraperitoneally for 8 days significantly increased serum creatinine. Serum creatinine concentration is a more potent indicator than the urea in the first phases of kidney disease. Furthermore, urea concentrations begin to increase only after parenchyma injury (Erdem et al., 2000). The ability of the kidney to filter creatinine (a non-protein waste product of creatinine phosphate metabolism) is reduced during renal dysfunction as a result of diminnished GFR. Thus, the increase in serum creatinine level is an indication of renal dysfunction (Perrrone et al., 1992).

In the present study, gentamicin pre or posttreatment with CoQ10 resulted in significant reduction in the elevated levels of serum creatinine and urea. This is in agreement with Fouad et al. (2010) who found that CoQ10 significantly reduced BUN and serum creatinine levels which were increased by cisplatin. The success of CoQ10 in reducing serum creatinine and urea concentrations could be attributed to its antioxidant properties because it has been found that ROS may be involved in the impairment of GFR (Pedraza-Chaverri et al., 2000). This is also concordant with the reports of Brunton et al. (2006) who stated that the impairment in renal function is almost always reversible because the proximal tubular cells have the capacity to regenerate. The apparent protective effect might be due to the ability of CoQ10 to neutralize the increase in free radicals caused by gentamicin.

The present results indicated that treatment with gentamicin alone resulted in atrophy in glomeruli, widening in Bowman's capsule, tubular dilatation and desquamation of their lining cells, interstitial hemorrhage, tubular necrosis and congested blood vessels. Cellular infiltration and hyaline degeneration were also showed. These changes were most prominent in the proximal convoluted tubules. In gentamicin pre or post-treated with CoQ10 groups, the renal corpuscles and tubules appeared more or less like normal.

Histological sections of renal cortex of gentamicin treated mice showed tubular dilatation with desquamation of their cells and even complete loss of lining cells of some tubules. Similar findings were observed by Al-Majed et al. (2002) who found that treatment with gentamicin (80 $\mathrm{mg} / \mathrm{kg} /$ day, intraperitoneally) for 8 days resulted in necrosis and desquamation of tubular epithelial cells in renal cortex as well as interstitial nephritis.

In the present study, congested blood vessels with foci of interstitial hemorrhage were also shown in group that treated with gentamicin alone. These results are in accordance with Shaday et al. (2007) who found that rats that injected intraperitoneally with $100 \mathrm{mg} / \mathrm{kg}$ b.w. of gentamicin showed congested blood vessels and interstitial hemorrhage. This also agreed with Ysebaert et al., (2000) and Sekhon et al., (2003).

Gentamicin only treated group also showed cellular degeneration and desquamation. These results are confirmed with Nasri et al. (2013) who reported that administration of gentamicin (80 mg/kg) for 10 days caused degeneration of the renal cells and tubular damage. These changes could be referred to renal ischemia caused by the toxic effect of gentamicin on renal blood vessels as previously explained by Mazzon et al. (2001) who stated that toxic renal damage may cause tubular injury, leading to the generation of oxygen free radicals, which are considered as important modulators of renal blood flow. Servais et al. (2006) found that gentamicin enter proximal tubular cells by pinocytosis from the luminal pole and accumulate in lysosomes and causes apoptosis at clinically relevant doses. This agreed with Han et al. (2006) who stated that the neprotoxicity may be closely associated with activation of proapoptotic proteins.

The tubular necrosis and degenerative changes seen in the present work conform with the findings of previous investigations (Dehghani et al., 2011). The results of the present work showed that the cortex of the kidney was more affected than the medulla as a result of long-term treatment with gentamicin. This might indicate that a relatively higher concentration of gentamicin reaches the cortex via the bloodstream than that entering the medulla. This is in agreement with the findings of Karahan et al. (2005) who stated that most of the gentamicin accumulates in the renal cortex. 
The current study also observed that treatment with gentamicin alone resulted in cellular infiltrations considered, as a prominent response of the body tissue facing any injurious impacts. These modifications could be due to the accumulation of free radicals resulting from an increased lipid peroxidation in the renal tissues of the gentamicin-treated group. Similar changes were also reported by Kumar et al. (2000) and Stojiljkovic et al. (2009) who demonstrated structural changes in renal tissue of gentamicin-treated animals and its reversal by various agents.

In the present study, gentamicin groups that pre or post-treated with CoQ10, in general, had less histological abnormalities and greater renal function than the mice given the same dose of gentamicin but without CoQ10, suggesting that CoQ10 does not adversely affect the kidney. These findings are supported by El-Sheikh et al. (2012) and Persson et al. (2012). Also, Pedraza-Chaverri et al. (2003) and Mehri et al. (2005) reported that the addition of antioxidants with aminoglycosides therapy attenuates the renal damage and protects the kidney from the oxidative degenerative changes. On the other hand, Sutken et al. (2007) demonstrated that CoQ10 has no protective effect against nephrotoxicity that induced by ochratoxin in kidney tissue. In conclusion, the present study suggests that CoQ10 has a renal protective potential. The nephroprotective effect of CoQ10 against gentamicininduced renal toxicity may be ascribed to its anti-oxidant, anti-apoptosis and anti-inflammatory properties.

\section{References}

Al-Majed AA, Mostafa AM, Al-Rikabi AC and AlShabanah OA (2002). Protective effects of oral arabic gum administration on gentamicin-induced nephrotoxicity in rats. Pharmacol Res. 46: 445-51.

Brunton LL, Lazo GS and Parker KL (2006). In : Goodman \& Gilman's. The Pharmacological Basis of Therapeutics. Mc Graw-Hill Inc, $11^{\text {th }}$ ed. PP.1155- 1167.

Cuzzocrea S, Mazzon E, Dugo L, Serraino I, Di Paola R, Britti D, De Sarro A, Pierpaoli S, Caputi A, Masini E and Salvemini D (2002). A role for superoxide in gentamicin- mediated nephropathy in rats. Eur J Pharmacol. 450(1): 67-76.

Dehghani F, Namavar MR, Noorafshan A, KarbalayDoust S and Esmaeilpour T (2011). Evaluation of the kidney extract on gentamicin-induced nephrotoxicity in rat. Kidney Res J. 1(1): 24-32.

Drury RA and Wallington EA (1980). Carleton's Histological Techniques, $5^{\text {th }}$ ed. Oxford: Oxford University Press.p. 362

El-Sheikh AA, Morsy MA, Mahmoud MM, Rifaai RA and Abdelrahman AM (2012). Effect of coenzyme-q10 on Doxorubicin-induced nephrotoxicity in rats. Adv Pharmacol Sci. 2012: 981461.

Erdem A, Gundogan NU, Usubutun A, Kilinc K, Erdem SR, Kara A and Bozkurt A (2000). The protective effect of taurine against gentamicin-induced acute tubular necrosis in rats. Nephrol Dial Transplant. 15: 1175-1182.
Farombi EO and Ekor M (2006). Curcumin attenuates gentamicin induced renal oxidative damage in rats. Food Chem Toxicol. 44: 1443-1448.

Faulkner WP and King JW (1976). Fundamentals of clinical chemistry $2^{\text {nd }}$ ed. Saunders Company, Philadelphia, USA. pp. 994-998.

Fouad AA, Al-Sultan AI, Refaie SM and Yacoubi MT (2010). Coenzyme Q10 treatment ameliorates acute cisplatin nephrotoxicity in mice. Toxicology. 274(1-3): 4956.

Han SY, Chang EJ, Choi H J, Kwak CS, Park SB, Kim HC and Mun KC (2006). Apoptosis by cyclosporine in mesangial cells. Transplant Proc. 38(7): 2244-6.

Kalayarasan S, Prabhu PN, Sriram N, Manikandan R, Arumugam M and Sudhandiran G. (2009). Diallyl sulfide enhances antioxidants and inhibits inflammation through the activation of Nrf2 against gentamicin-induced nephrotoxicity in Wistar rats. Eur J Pharmacol. 606: 162171.

Karadeniz A, Yildirim A, Simsek N, Kalkan Y and Celebi F (2008). Spirulina platensis protects against gentamicin-induced nephrotoxicity in rats. Phytothe Res. 22: 1506-1510.

Karahan I, Ateşşahin A, Yilmaz S, Ceribaşi AO and Sakin F (2005). Protective effect of lycopene on gentamicin-induced oxidative and nephrotoxicity in rats. Toxicology. 215:198-204.

Khan SA, Priyamvada S, Farooq N, Khan S, Khan MW and Yusufi AN (2009). Protective effect of green tea extract on gentamicin-induced nephrotoxicity and oxidative damage in rat kidney. Pharmacol Res. 59: 254-62.

Kumar C, Himabindu M and Jetty A (2008). Microbial biosynthesis and applications of gentamicin: a critical appraisal. Crit Rev Biotech. 28:173-212.

Littarru GP and Tiano L (2007). Bioenergetic and antioxidant properties of coenzyme Q10: recent developments. Mol Biotechnol. 37(1): 31-7.

Martínez-Salgado C, Henández-López, FJ and Novoa-López JM (2007). Glomerular nephrotoxicity of aminoglycosides. Toxicol Appl Pharmacol. 223(1): 86-98.

Mehri K, Hossein K, Mahdieh F, Rana G and Maryam Z (2005). Effects of co-supplementation of vitamins $E$ and $C$ on gentamicin-induced nephrotoxicity in rats. Exp Physiol. 90(4): 571-576.

Mizrak B, Parlakpinar H, Acet A and Turkoz Y

(2004). Effects of pinealectomy and exogenous melatonin on rat hearts. Acta Histochem. 106: 29-36.

Moreira MA, Nascimento MA, Bozzo TA, Cintra A, da Silva SM, Dalboni MA, Mouro MG and Higa E.M.

(2014). Ascorbic acid reduces gentamicin-induced nephrotoxicity in rats through the control of reactive oxygen species. Clin Nutr. 33: 296-301.

Negrette-Guzmán M, Huerta-Yepez S, MedinaCampos ON, Zatarain-Barrón ZL, Hernández-Pando R, Torres I, Tapia E and Pedraza-Chaverri J (2013). Sulforaphane attenuates gentamicin-induced nephrotoxicity: role of mitochondrial protection. Evidance Based Complement.Alt.Med. 2013: 1-17. 
Parlakpinar H, Tasdemir S, Polat A, Bay-karabulut A, Vardi N, Ucar M and Acet A (2005). Protective role of caffeic acid phenethyl ester (cape) on gentamicin-induced acute renal toxicity in rats. Toxicol. 207: 169-77.

Patton CJ and Crouch SR (1977). Spectrophotometric and kinetics investigation of the Berthelot reaction for the determination of ammonia: Analytical Chemistry. 49: 464-469.

Pedraza-Chaverrí J, González-Orozco AE, Maldonado PD, Barrera D, Medina-Campos ON and Hernández-Pando R (2003). Diallyl disulfide ameliorates gentamicin-induced oxidative stress and nephropathy in rats. Eur J Pharmacol. 473: 71-78.

Pedraza-Chaverrí J, Maldonado PD, MedinaCampos ON, Olivares-Corichi IM, Granados-Silvestre MA, Hernández-Pando R and Ibarra-Rubio ME (2000). Garlic ameliorates gentamicin nephrotoxicity: relation to antioxidant enzymes. Free Radic Biol Med. 29: 602-611.

Perrrone RD, Madias NE and Levey AS (1992). Serum creatinine as an index of renal function: new insights into old concepts. Clin Chem. 38: 1933-53.

Persson MF, Franzén S, Catrina SB, Dallner G, Hansell P, Brismar K and Palm F (2012). Coenzyme Q10 prevents GDP-sensitive mitochondrial uncoupling, glomerular hyperfiltration and proteinuria in kidneys from $\mathrm{db} / \mathrm{db}$ mice as a model of type 2 diabetes. Diabetologia. 55(5): 1535-43.

Reiter RJ, Tan DX, Sainz RM, Mayo JC and LopezBurillo S (2002). Melatonin: reducing the toxicity and increasing the efficacy of drugs. J Pharm Pharmacol. 54: 1299-321.

Sahna E, Parlakpinar H, Ozturk F, Cigremis Y and Acet A (2003). The protective effects of physiological and pharmacological concentrations of melatonin on renal ischemiareperfusion injury in rats. Urol Res. 31: 188-93.

Sanford HS (1954). Method for obtaining venous blood from orbital sinus of the rat or mouse. science. 119: $100-102$.
Sekhon CS, Sekhon BK, Singh I, Orak JK and Singh AK (2003). Attenuation of renal ischemia/reperfusion injury by a triple drug combination therapy. J Nephrol. 16: 63-74.

Servais H, Jossin Y, Van Bambeke F, Tulkens PM and Mingeot-Leclercq MP (2006). Gentamicin causes apoptosis at low concentrations in renal LLC-PK1 cells subjected to electroporation. Antimicrob Agents Chemother. 50: 1213-1221.

Shaday AM, Zeedan OI and El-Saify GH (2007). Ultrastructural \& Immunohistochemical Study On The Effect Of The Antioxidant N-Acetylcystein On Gentamycin-Induced Renal Cortical Damage In Adult Male Albino Rats. Menoufiya Med J. 20 (2): 193-204.

Stojiljkovic N, Veljkovic S, Mihailovic D, Stoiljkovic M, Radenkovic M, Rankovic G and Randjelovic P (2009). Protective effects of pentoxifylline treatment on gentamicin-induced nephrotoxicity in rats. Ren Fail. 31(1): pp. 54-61.

Sun X, Zhang B, Hong X, Zhang X and Kong X. (2013). Histone deacetylase inhibitor, sodium butyrate, attenuates gentamicin-induced nephrotoxicity by increasing prohibitin protein expression in rats. Eur J Pharmacol. 707: 147-154.

Sutken E, Aral E, Ozdemir F, Uslu S, Alatas O and Colak O (2007). Protective role of melatonin and coenzyme Q10 in ochratoxin A toxicity in rat liver and kidney. Int J Toxicol. 26(1): 81-7.

Villalba JM, Parrado C, Santos-Gonzalez $M$ and Alcain FJ (2010). Therapeutic use of coenzyme Q10 and coenzyme Q10-related compounds and formulations. Expert Opin Investig Drugs. 19(4): 535-554.

Ysebaert DK, De Greef KE, Vercauteren SR, Ghielli M, Verpooten GA, Eyskens EJ and De Broe ME (2000). Identification and kinetics of leukocytes after severe ischaemia/reperfusion renal injury. Nephrol Dial Transplant. 15: 1562-1574. 